\title{
Investigation of retained strains and microstructures for a hot deformed stainless steel
}

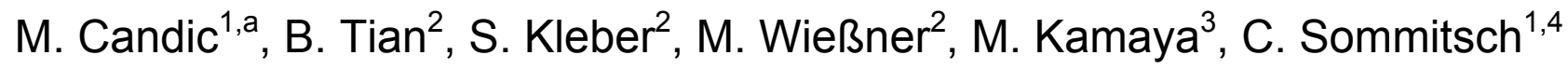 \\ ${ }^{1}$ Christian Doppler Laboratory for Materials Modelling and Simulation, Franz-Josef-Str.18, \\ A-8700 Leoben, Austria
}

${ }^{2}$ Böhler Edelstahl GmbH \& Co KG, P.O. Box 96, A-8605 Kapfenberg, Austria
${ }^{3}$ Institute for Nuclear Safety Sytem, Inc., Nuclear Power Plant Aging Research Center, 64 Sata, Mihama-cho, Mikata-gun, Fakui 919-1205, Japan

${ }^{4}$ Chair of Metal Forming, University of Leoben, Franz-Josef-Str. 18, A-8700 Leoben, Austria

amirza.candic@cdl-mms.at

Keywords: retained strain, hot deformation, recrystallization, 304L

\begin{abstract}
For the investigation of retained strains during hot forming, which are related to the dislocation structure, single and double hit compression tests were carried out at different temperatures and strain rates for a stainless steel. Using microhardness measurements the retained strains after the first and second pass were investigated as a function of the amount of deformation, temperatures as well as strain rates and dwell durations. In general, the retained strain decreases with increasing dwell durations in both the deformed and recrystallized grains, respectively. At a given total amount of deformation in a double hit compression, the retained strains for the as deformed unrecrystallized grains are reduced for a lower deformation in the first hit. For the recrystallized grains the retained strain increases, when comparing double hit with single hit compression.
\end{abstract}

\section{Introduction}

For the hot forming processes, retained strains exist due to inhomogenous deformation as well as different degrees of recovery and recrystallization, which are furthermore related to both strain rate and temperature gradients during hot forming. The retained strains are in the following related to the stored deformation energy, i.e. dislocation structure. Deformation energy is reduced during a subsequent annealing, which may lead to abnormal grain growth and hence to an inhomogenous grain structure [1].

For industrial processes, the strain, accumulated over all passes, can be calculated by the finite element method (FEM). However, the obtained effective strain is unreallistically high for multipass hot deformation if the changes in microstructure (recovery, recrystallization) are not included in the calculations. For a multipass deformation process the dislocation density is reduced due to the occurance of recovery and rycrstallization during and after the first deformation pass, thus the strain retained for the next pass of deformation should cover a range between zero and the accumulated strain. The retained strain is a critical parameter not only for an accurate prediction of the working loads, but also for the applications in microstructure modelling.

The aim of the present work was to study the development of the retained strain during double hit compression of the austenitic stainless steel 304L, which deformation behaviour has been studied elsewhere [1-5]. Microhardness measurements were carried out in order to investigate retained strains as well as their distribution. The misorientations of the substructures were determined by EBSD and TEM analyses. 


\section{Experimental procedure}

A 304L-type austenitic stainless steel (Böhler A607, Fe-19Cr-9Ni), with an initial grain size of about $20 \mu \mathrm{m}$, was homogenized at $1200^{\circ} \mathrm{C}$ for 20 minutes, afterwards deformed by compression in a Gleeble $3800^{\mathrm{TM}}$ system and subsequently air cooled to room temperature. After homogenization an initial grain size of about $50 \mu \mathrm{m}$ was reached.

Hot deformation was performed at two different conditions, which aimed firstly a deformed unrecrystallized grain structure $\left(\mathrm{T}_{\mathrm{def}}=750^{\circ} \mathrm{C}\right.$, strain rate $\left.=1.0 \mathrm{~s}^{-1}\right)$ and secondly a fully recrystallized grain structure $\left(\mathrm{T}_{\text {def }}=1050^{\circ} \mathrm{C}\right.$, strain rate $\left.=0.1 \mathrm{~s}^{-1}\right)$. Dwell times $\left(\mathrm{t}_{\mathrm{d}}\right)$ varied between 3 and 10 seconds. Following hot deformation, the specimens were cut perpendicular to the tensile axis and the microhardness measurements $\left(\mathrm{HV}_{0.980}\right)$ were carried out in the center of deformed samples, where the local strains have been determined by the finite element calculations. The grain structures were investigated using conventional optical microscope and for the confirmation of the obtained results EBSD measurements and TEM investigations were used.

\section{Results and Discussion}

Changes of retained strains due to single and double hit compression. The microhardness (HV) is proportional to the actual strain $\left(\varphi_{\text {actual }}\right)$ and further to the dislocation density $(\rho)$, which can be stated as follows:

$H V \propto \varphi_{\text {actual }} \propto \rho$

For the deformed grains without any recrystallization, the retained strain $\left(\varphi_{\text {retained }}^{\text {def }}\right)$ was defined using the following equation:

$\varphi_{\text {retained }}^{\text {def }}=\frac{H V^{\left(d w e l l_{-} \text {time } \neq 0\right)}-H V_{(\varphi=0)}}{H V^{\left(d w e l l_{-} \text {time }=0\right)}-H V_{(\varphi=0)}} \varphi_{e q}$

with

$\mathrm{HV}_{(\varphi=0)} \quad$ : microhardness of the homogenized un-deformed specimen,

$\mathrm{HV}^{(\mathrm{dwell} \text { time } \neq 0)}$ : microhardness after deformation and a dwell time $\left(\mathrm{t}=\mathrm{t}_{\mathrm{d}}\right)$,

$\mathrm{HV}^{(\mathrm{dwell} \text { time }=0)}$ : microhardness after deformation without dwell $\left(\mathrm{t}_{\mathrm{d}}=0\right)$. When $\mathrm{HV}^{(\mathrm{t}=\mathrm{td})}=\mathrm{HV}^{(\mathrm{td}=0)}$, the

retained strain ( $\left.\varphi_{\text {retained }}\right)$ coincides with the equivalent (i.e. accumulated) strain $\left(\varphi_{\mathrm{eq}}\right)$,

$\varphi_{\text {eq }}$ : local equivalent strain calculated by FEM.

During dynamic recrystallization, the recrystallized grains experience a subsequent deformation until a second recrystallization cycle starts [6]. Hence at an arbitrary strain that is higher than the critical strain for the onset of dynamic recrystallization $\varphi_{\mathrm{cr}}$, the strain distribution within all recrystallized grains is between 0 and $\varphi_{\text {cr }}$. Regardless of the microstructure difference in each single grain, the maximum strain that can be achieved within a recrystallized grain cannot be beyond the critical strain for dynamic recrystallization. Therefore, the maximum retained strain is $\varphi_{c r}$ for a single hit compression. During a dwell period after the compression, the stored deformation energy in the recrystallized grains is reduced by static recovery. The retained strain is assumed to be zero if the grain structure is recovered completely. In the present work it is assumed that the recovery is complete at $t_{d}=10$ s under the given process conditions. The retained strain for the recrystallized grains $\left(\varphi_{\text {retained }}^{\text {rex }}\right)$ can be defined as follows: 
$\varphi_{\text {retained }}^{r e x}=\frac{H V^{\left(d w e l l_{-} \text {time } \neq 0\right)}-H V_{(\text {dwell_time }=10 s)}}{H V^{\left.(\text {dwell_time }=0)_{-}\right)}-H V_{(\text {dwell_time }=10 s))}} \varphi_{c r}$

with

$\mathrm{HV}^{(\mathrm{dwell} \text { time }=0)}:$ microhardness for the recrystallized grains at $\mathrm{t}_{\mathrm{d}}=0$,

$\mathrm{HV}^{(\mathrm{dwell} \text { time } \neq 0)}:$ microhardness for the recrystallized grains at a given dwell duration $\left(\mathrm{t}=\mathrm{t}_{\mathrm{d}}\right)$,

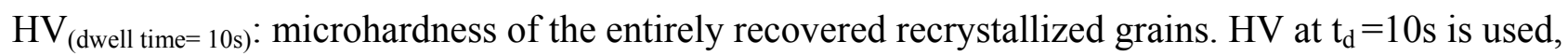

$\varphi_{\mathrm{cr}}$ : critical strain for dynamic recrystallization, which is determined from the peak strain of the flow stress curve. $\varphi_{\mathrm{cr}}=0.80 \varphi_{\text {peak }}[7]$. Hence follows $\varphi_{\mathrm{cr}}=0.24$ for $304 \mathrm{~L}$.

The comparison of the retained strains during a single hit compression for the deformed (unrecrystallized) grains and for completely recrystallized grains is shown in Fig. 1. Therefore, for the single hit compression the retained strain decreases with dwell duration both in deformed (unrecrystallized) and recrystallized grains. For the unrecrystallized grains a higher retained strain is expected with a higher amount of deformation, but for the recrystallized grains, regardless of the applied strain, after a dwell time of 10 s, the recovery is almost completed.

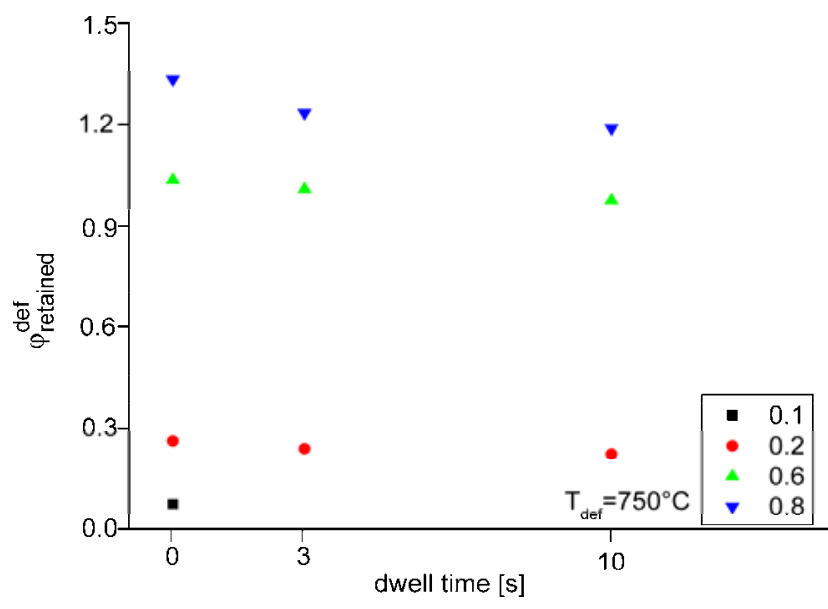

(a)

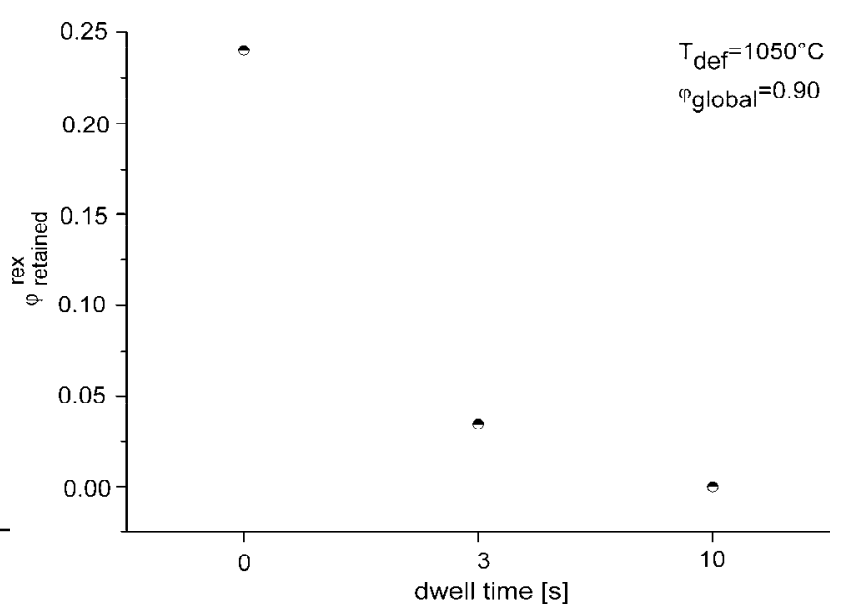

(b)

Fig. 1: Comparison of local retained strains in a single hit compression after different dwell times for the austenitic stainless steel 304L in (a) deformed (unrecrystallized) grains and (b) recrystallized grains. The symbols correspond to the applied global strain (e.g. 0.60: the strain of the first hit is $\varphi_{1}=0.60$ ).

The comparison of the retained strains between single and double hit compression for unrecrystallized and recrystallized grains is shown in Fig. 2. For the recrystallized grains (Fig. 2b), the retained strains with long dwell times (here 10s) increase for a double hit compression comparing it with the single hit tests for the same total amount of deformation. It is assumed that during the dwell between the two hits a substructure, i.e. subgrains, is formed by recovery of dislocations in the recrystallized grains. This substructure leads to higher dislocation formation rates during the second deformation hit, which caused higher retained strains after the double hit compression tests. 


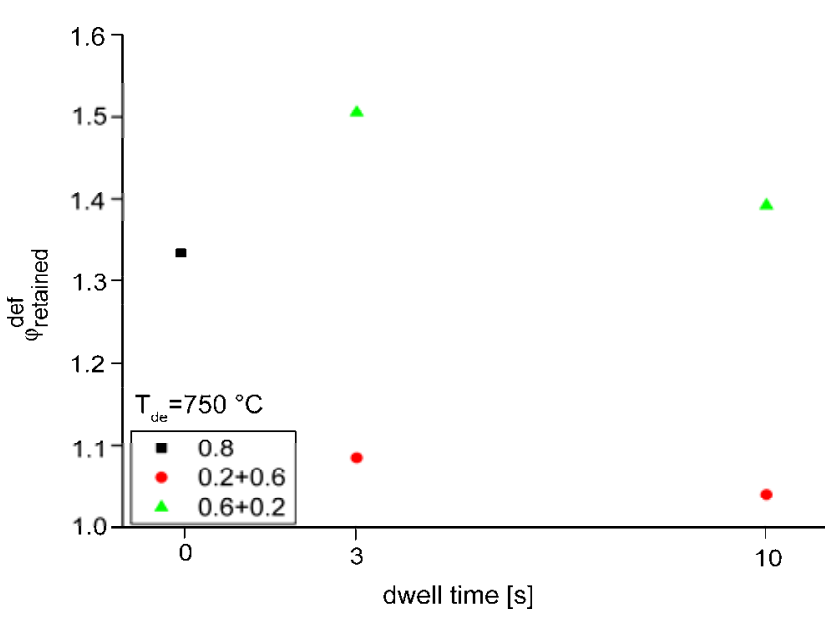

(a)

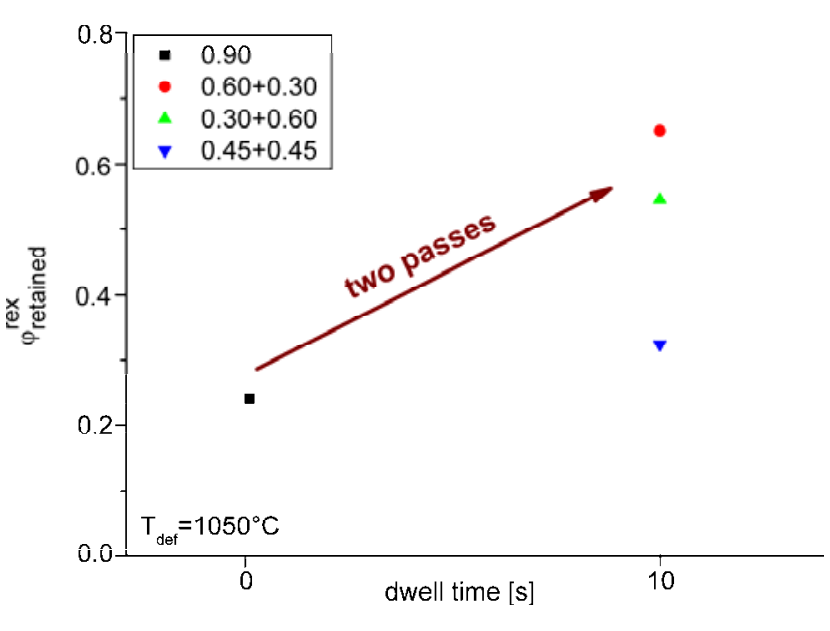

(b)

Fig. 2: Comparison of local retained strains in a single and double hit compression for the austentic stainless steel 304L in (a) deformed (unrecrystallized) grains and (b) recrystallized grains. The symbols correspond to the applied global strains of the first and second hit, respectively (e.g. $0.60+0.20$ : the global strain of the first hit is $\varphi_{1}=0.60$ the global strain of the second hit is $\varphi_{2}=0.20$ ).

For the deformed, unrecrystallized, grains (Fig. 2a) the retained strain during a double hit compression decreases with increasing dwell time. Comparing double hit with single hit compression tests, the retained strain increases for a higher deformation in the first hit. For relatively small deformations in the first hit, no substructures are formed during the dwell hence follows, smaller retained strains with the same total deformation can be expected.

Misorientations determined by EBSD and TEM investigations. In order to confirm the evaluated retained strain by microhardness measururments, some EBSD analyses were carried out. Therefore the MCD value (modified crystal deformation) was calculated, which quantifies the spread of the crystal orientation [8]. The evaluated parameter is defined as an average of the lognormal distribution of the misorientation from the central orientation of each grain.

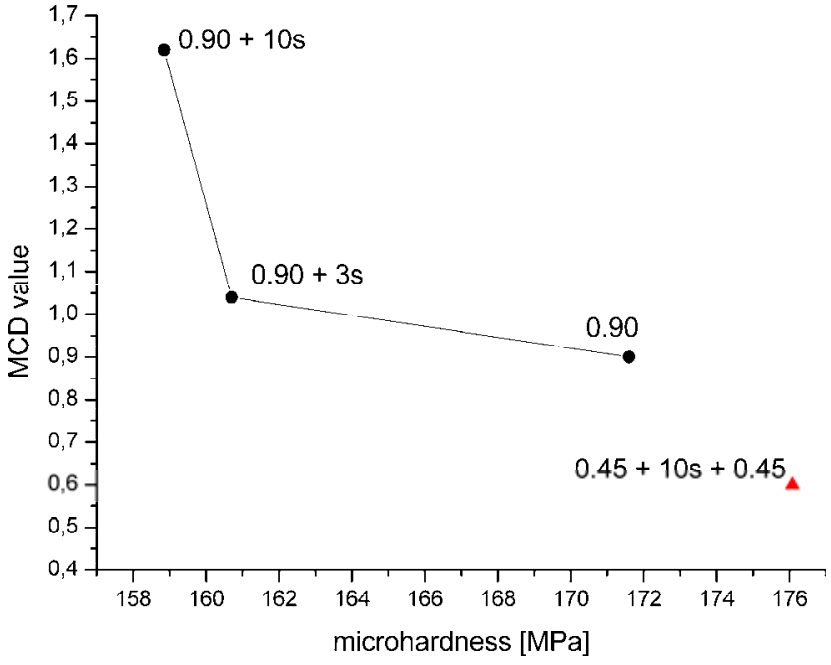

(a)

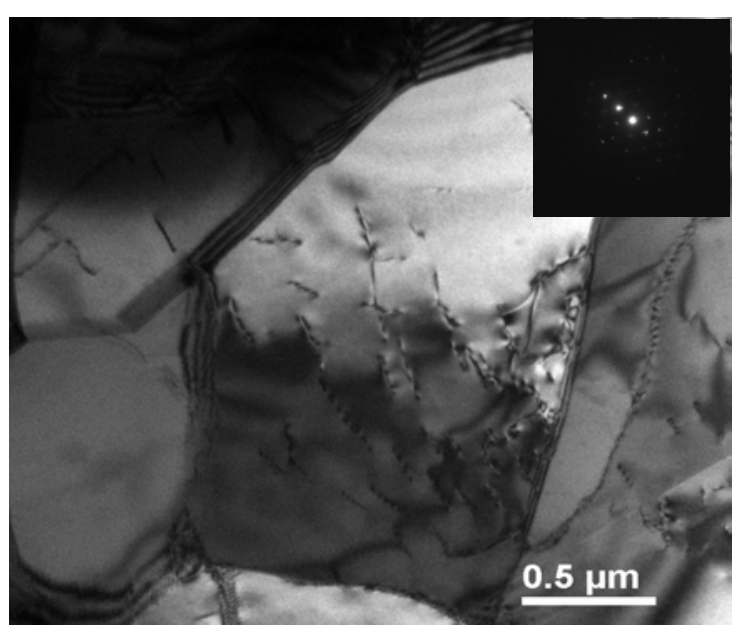

(b)

Fig. 3: The investigation of substructures, by (a) EBSD measurements and (b) subgrains observed by TEM for sample $0.45+10 \mathrm{~s}+0.45$. 
As assumed to be proportional to the dislocation density, $\mathrm{HV}$ and thus retained strain values inversely correspond to the modified crystal deformation values for both single and double hit compression (Fig. 3a compared with Fig. 1b and Fig. 2b). Figure 3b depicts the formed subgrain structure within a recrystallized grain after a double hit comparison.

\section{Summary}

For an austenitic stainless steel, the retained strain have been evaluated by means of microhardness measurements in unrcrystallized and recrystallized grains. The retained strain decreased with dwell durations both in deformed (unrecrystallized) and recrystallized grains.

At the same amount of total deformation, the retained strains were compared between single and double hit compression tests. For the recrystallized grains, the retained strains were increased due to the formation of a substructure during the dwell. The retained strains for the deformed unrecrystallized grains increased for a higher deformation in the first hit. However, due to recovery after a small deformation in the first hit, the retained strain after a double hit compression falls below the strain obtained after a single hit compression.

\section{References}

[1] J. Mizera, J.W. Wyrzykowski, K.J. Kurzydlowski: Mater. Sci. and Eng. 104A (1998), p. 157

[2] A. Belyakov, H. Miura, T. Sakai: Mater. Sci. and Eng. A255 (1998), p. 139

[3] S.H. Cho, Y.C. Yoo: J. Mater. Sci. 36 (2001), p. 4273

[4] A. Belyakov, K. Tsuzaki, H. Miura, T. Sakai: Mater. Sci. Forum 426-432 (2003), p. 1005

[5] S.H. Lee, J.S. Choi, D.Y. Yoon: Z. Metallk. 92 (2001), p. 655

[6] C. Sommitsch, W. Mitter: Acta Mater. 54 (2006), p. 357

[7] G.R. Stewart, J.J. Jonas, F. Montheillet: ISIJ Int. 44 (2004), p. 1581

[8] M. Kamaya, A.J. Wilkinson, J.M. Titchmarsh: Acta Mater. 54 (2006), p. 539 